\title{
Article \\ Hybrid Inertial Accelerated Algorithms for Solving Split Equilibrium and Fixed Point Problems
}

\author{
Yanlai Song
}

check for updates

Citation: Song, Y. Hybrid Inertial Accelerated Algorithms for Solving Split Equilibrium and Fixed Point Problems. Mathematics 2021, 9, 2680. https://doi.org/10.3390/math9212680

Academic Editor: Christopher Goodrich

Received: 9 September 2021 Accepted: 15 October 2021 Published: 22 October 2021

Publisher's Note: MDPI stays neutral with regard to jurisdictional claims in published maps and institutional affiliations.

Copyright: (c) 2021 by the authors. Licensee MDPI, Basel, Switzerland. This article is an open access article distributed under the terms and conditions of the Creative Commons Attribution (CC BY) license (https:/ / creativecommons.org/licenses/by/ $4.0 /)$.
College of Science, Zhongyuan University of Technology, Zhengzhou 450007, China; 6392@zut.edu.cn

\begin{abstract}
In this paper, we introduce a new hybrid inertial accelerated algorithm with a line search technique for solving fixed point problems for demimetric mapping and split equilibrium problems in Hilbert spaces. The algorithm is inspired by Tseng's extragradient method and the viscosity method. Then, we establish and prove the strong convergence theorem under proper conditions. Furthermore, we also give a numerical example to support the main results. The main results are new and the proofs are relatively simple and different from those in early and recent literature.
\end{abstract}

Keywords: strong convergence; split equilibrium problem; demimetric mapping; hybrid inertial accelerated algorithms; Armijo-like step size rule

\section{Introduction}

Let $C$ be a nonempty, closed, and convex subset of a real Hilbert space $\mathscr{H}$ with scalar product $\langle\cdot, \cdot\rangle$ and generated norm $\|\cdot\|$. Let $F: C \times C \rightarrow \mathbb{R}$ be a bifunction. The Equilibrium Problem (EP) is of the form

$$
\text { find a point } u \in C \text { such that } F(u, v) \geq 0, \forall v \in C \text {. }
$$

Denote the set of solutions of the $\mathrm{EP}$ as $\operatorname{EP}(F)$. The EP unifies many mathematical models such as the saddle point problems, the variational inequalities, and the optimization problems; see [1-5].

Given a mapping $B: C \rightarrow \mathscr{H}$, the Variational Inequality Problem (VIP) obtains a point $x^{*} \in C$ such that

$$
\left\langle B x^{*}, x-x^{*}\right\rangle \geq 0, \quad \forall x \in C .
$$

Solutions to these classes of problems, fixed point problems, and related optimization problems have been investigated and iterative algorithms for approximating them have been proposed and studied by several authors; see [6-9].

Let $\mathscr{H}_{1}$ and $\mathscr{H}_{2}$ be real Hilbert spaces, and let $C \subseteq \mathscr{H}_{1}$ and, $Q \subseteq \mathscr{H}_{2}$ be nonempty closed convex subsets. Let $F_{1}: C \times C \rightarrow \mathbb{R}$ and $F_{2}: Q \times Q \rightarrow \mathbb{R}$ be bifunctions. The Split Equilibrium Problem (SEP) finds

$$
z \in C \text { such that } z \in \operatorname{EP}\left(F_{1}\right) \bigcap A^{-1}\left(\operatorname{EP}\left(F_{2}\right)\right),
$$

where $A: \mathscr{H}_{1} \rightarrow \mathscr{H}_{2}$ is a bounded linear operator. Denote the set of solutions of the SEP as $\operatorname{SEP}\left(F_{1}, F_{2}\right)$. As we know, the SEP is a generalization of the EP by putting $F_{2}=0$ and $Q=\mathscr{H}_{2}$. The SEP has been found to be useful in the study of many real-world problems such as data compression, sensor networks, medical reconstructions, etc. Additionally, the SEP is an extension of many mathematical models such as the split inclusion problem, the split feasibility problems, and the split variational inequality problems (SVIP, in which the solution set is denoted by $\operatorname{SVIP}(C, Q))$; see [10-12] and the references therein. 
In order to find a common solution for the fixed point problem for nonexpansive mapping and the SEP, Kazmi and Rizvi [12] suggested the following projection algorithm:

$$
\left\{\begin{array}{l}
x_{n+1}=\alpha_{n} u+\beta_{n} x_{n}+\gamma_{n} S y_{n}, \\
y_{n}=P_{C}\left(u_{n}-\lambda_{n} T u_{n}\right), \\
u_{n}=T_{r_{n}}^{F_{1}}\left(x_{n}-\gamma A^{*}\left(I-T_{r_{n}}^{F_{2}}\right) A x_{n}\right), n \geq 1
\end{array}\right.
$$

where $S: C \rightarrow C$ is nonexpansive and $T: C \rightarrow \mathscr{H}_{1}$ is inverse strongly monotone. Then, the sequence $\left\{x_{n}\right\}$ converges strongly to an element in the solution set.

Recently, Jolaoso and Karahan [13] proposed the following general alternative regularization algorithm (Algorithm 1) for approximating a solution of the SEP and the fixed point for demicontractive mapping:

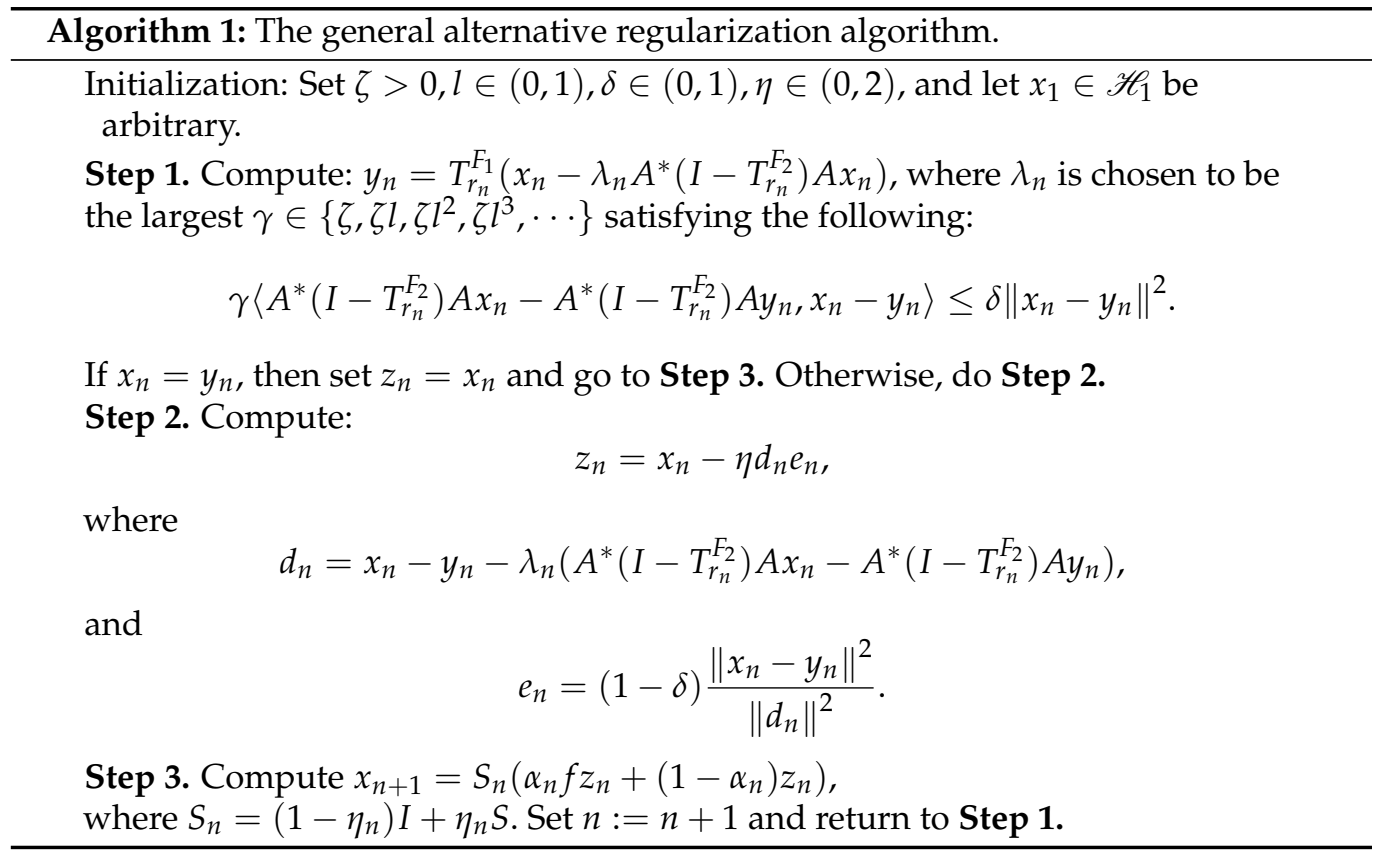

Assuming that $S: \mathscr{H}_{1} \rightarrow \mathscr{H}_{1}$ is a $k$-demicontractive mapping, $A: \mathscr{H}_{1} \rightarrow \mathscr{H}_{2}$ is a bounded linear operator with adjoint $A^{*}: \mathscr{H}_{2} \rightarrow \mathscr{H}_{1}, f: \mathscr{H}_{1} \rightarrow \mathscr{H}_{1}$ is Lipschitz and strongly pseudo-contractive, $\eta_{n} \in(0,1)$, and $0<\liminf _{n \rightarrow \infty} \eta_{n} \leq \limsup _{n \rightarrow \infty} \delta_{n}<1-k$. Then, under certain appropriate assumptions, Algorithm 1 is to proven to converge strongly to the unique element.

On the other hand, in 2001, Alvarez and Attouch [14] used the heavy ball method that was studied in $[15,16]$ for maximal monotone operators on the proximal point algorithm. The algorithm is said to be the inertial proximal point algorithm and it is as follows:

$$
\left\{\begin{array}{l}
x_{n+1}=\left(I+r_{n} T\right)^{-1} y_{n}, \\
y_{n}=x_{n}+\theta_{n}\left(x_{n}-x_{n-1}\right), n \geq 1 .
\end{array}\right.
$$

They also proved that the sequence $\left\{x_{n}\right\}$ constructed by (1) converges weakly to a zero of $T$ under some suitable conditions.

Moreover, Cai et al. [17] proposed the following modified Tseng's extragradient (Algorithm 2) for solving the pseudo-monotone variational inequality problem: 


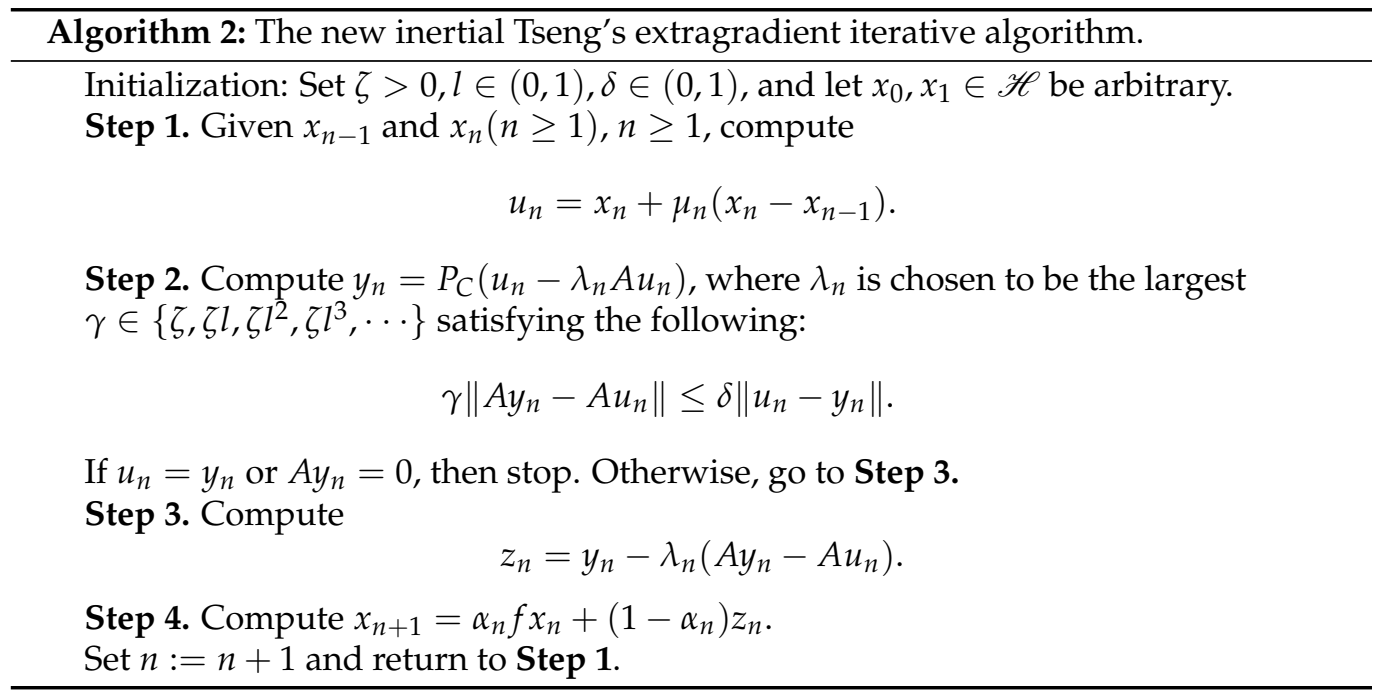

Assume that $f: \mathscr{H} \rightarrow \mathscr{H}$ is contractive, $\left\{\alpha_{n}\right\} \subset(0,1)$, and $\left\{\mu_{n}\right\}$ is a sequence in $[0,1)$ satisfying $\lim _{n \rightarrow \infty} \frac{\mu_{n}}{\alpha_{n}}\left\|x_{n}-x_{n-1}\right\|=0$. Then, they proved that the iterative sequence $\left\{x_{n}\right\}$ generated by Algorithm 2 converges to a solution of the pseudo-monotone variational inequality problem under some suitable conditions.

Motivated and inspired by Kazmi and Rizvi [12], Jolaoso and Karahan [13], Alvarez and Attouch [14], and Cai et al. [17], we suggest and analyze a hybrid inertial accelerated method for finding a common element of the set of fixed points of a demimetric mapping and the set of solutions of the split equilibrium problem in a real Hilbert space. We then prove a strong convergence of the proposed method under some mild conditions. Finally, we also provide a numerical experiment to demonstrate the efficiency of the proposed method over some existing ones.

\section{Preliminaries}

Throughout this paper, we denote the set of fixed points of a mapping $T$ as $\operatorname{Fix}(T)$.

Lemma 1 ([18]). In a real Hilbert space $\mathscr{H}$, the following hold:

(1) $\|k u+(1-k) v\|^{2}=k\|u\|^{2}+(1-k)\|v\|^{2}-k(1-k)\|u-v\|^{2}, \forall u, v \in \mathscr{H}$ and $k \in[0,1]$;

(2) $\|u \pm v\|^{2}=\|u\|^{2} \pm 2\langle u, v\rangle+\|v\|^{2}, u, v \in \mathscr{H}$;

(3) $\|u+v\|^{2} \leq\|u\|^{2}+2\langle v, u+v\rangle, u, v \in \mathscr{H}$.

Lemma 2 ([19]). Let $C$ be a nonempty closed convex subset of a real Hilbert space $\mathscr{H}$. For $u \in \mathscr{H}$ and $v \in C, v=P_{C} u$ if and only if

$$
\langle u-v, w-v\rangle \leq 0, \quad \forall w \in C,
$$

where $P_{C}$ is the metric projection from $\mathscr{H}$ onto $C$.

Definition 1. A mapping $S: C \rightarrow \mathscr{H}$ is said to be

(1) nonexpansive, if

$$
\|S u-S v\| \leq\|u-v\|, \forall u, v \in C ;
$$

(2) $\gamma$-contractive, if there exists $\gamma \in[0,1)$ such that

$$
\|S u-S v\| \leq \gamma\|u-v\|, \forall u, v \in C ;
$$

(3) quasi-nonexpansive, if $\operatorname{Fix}(S) \neq \varnothing$ and

$$
\left\|S u-x^{*}\right\| \leq\left\|u-x^{*}\right\|, \forall u \in C, x^{*} \in \operatorname{Fix}(S) ;
$$


(4) $\alpha$-strongly pseudo-contractive, if there exists a constant $\alpha \in[0,1)$, such that

$$
\langle S u-S v, u-v\rangle \leq \alpha\|u-v\|^{2}, \quad \forall u, v \in C
$$

(5) pseudo-monotone, if

$$
\langle S v, u-v\rangle \geq 0 \Rightarrow\langle S u, u-v\rangle \geq 0, \quad \forall u, v \in C
$$

(6) $k$-demicontractive, if $\operatorname{Fix}(S) \neq \varnothing$ and there exists $k \in[0,1)$, such that

$$
\left\|S u-x^{*}\right\|^{2} \leq\left\|u-x^{*}\right\|^{2}+k\|u-S u\|^{2}, \forall u \in C, x^{*} \in \operatorname{Fix}(S) ;
$$

(7) $k$-demimetric, if $\operatorname{Fix}(S) \neq \varnothing$ and there exists $k \in(-\infty, 1)$, such that

$$
\left\|S u-x^{*}\right\|^{2} \leq\left\|u-x^{*}\right\|^{2}+k\|u-S u\|^{2}, \forall u \in C, x^{*} \in \operatorname{Fix}(S) .
$$

It is clear that the class of $k$-demimetric mappings contains the classes of $k$-demicontractive mappings. In 2017, Takahashi [20] also gave an example for a demimetric mapping that is not a demicontractive mapping, as follow:

Example 1 ([20]). Let $\mathscr{H}$ be a real Hilbert spaces and $C$ be a nonempty closed convex subsets of $\mathscr{H}$. Denote the metric projection of $\mathscr{H}$ onto $C$ as $P_{C}$. Then, $P_{C}$ is $(-1)$-demimetric but $P_{C}$ is not demicontractive.

Assume that $C$ is a nonempty, convex, and closed subset of a Hilbert space $\mathscr{H}$ and $F: C \times C \rightarrow \mathbb{R}$ is a bifunction satisfying the following restrictions:

(A1) $F(u, u)=0, \forall u \in C$;

(A2) $F$ is monotone, i.e., $F(u, v)+F(v, u) \leq 0, \forall u, v \in C$;

(A3) For all $u, v, w \in C$, $\lim \sup _{t \downarrow 0} F(t w+(1-t) u, v) \leq F(u, v)$;

(A4) For all $u \in C, F(u, \cdot)$ is convex and lower semicontinuous.

The following lemmas are needed.

Lemma 3 ([21]). Let $C$ be a nonempty closed convex subset of a real Hilbert space $\mathscr{H}$, and $T: C \rightarrow C$ be nonexpansive. Then, the mapping $I-T$ is demiclosed at zero, i.e., if $\left\{x_{n}\right\}$ converges weakly to a point $x \in C$ and $\left.\{I-T) x_{n}\right\}$ converges to zero; then, $x=T x$.

Lemma 4 ([22,23]). Suppose that $C$ is a nonempty close convex subset of a real Hilbert space $\mathscr{H}$. Assume that $S: C \rightarrow \mathscr{H}$ is $k$-demimetric such that $\operatorname{Fix}(S)$ is nonempty. Let $\kappa$ be a real number with $\kappa \in(0, \infty)$, and define $T=(1-\kappa) I+\kappa S$. Then, it holds that

(1) $\operatorname{Fix}(T)=\operatorname{Fix}(S)$ if $\kappa \neq 0$;

(2) $T$ is a quasi-nonexpansive mapping for $\kappa \in(0,1-k]$;

(3) $\operatorname{Fix}(S)$ is a closed convex subset of $\mathscr{H}$.

Lemma 5 ([24]). Assume that $\left\{a_{n}\right\}$ is a sequence of real numbers such that there exists a subsequence $\left\{n_{i}\right\}$ of $\{n\}$ with $a_{n_{i}}<a_{n_{i}+1}$ for all $i \in \mathbb{N}$. Then, there exists a nondecreasing sequence $\left\{m_{j}\right\} \subseteq \mathbb{N}$ such that $m_{j} \rightarrow \infty$ and the following properties are satisfied for all (sufficiently large) numbers $j \in \mathbb{N}$ :

$$
a_{m_{j}} \leq a_{m_{j}+1} \text { and } a_{j} \leq a_{m_{j}+1}
$$

Indeed, $m_{j}=\max \left\{k \leq j: a_{k}<a_{k+1}\right\}$.

Lemma 6 ([25]). Assume that $\left\{a_{n}\right\}$ is a sequence of nonnegative numbers satisfying the following inequality:

$$
a_{n+1} \leq\left(1-\beta_{n}\right) a_{n}+\gamma_{n}+\beta_{n} \delta_{n}, \quad n \in \mathbb{N}
$$


where $\left\{\beta_{n}\right\},\left\{\gamma_{n}\right\},\left\{\delta_{n}\right\}$ satisfy the following restrictions:

(i) $\sum_{n=1}^{\infty} \beta_{n}=\infty, \quad \lim _{n \rightarrow \infty} \beta_{n}=0$,

(ii) $\gamma_{n} \geq 0, \quad \sum_{n=1}^{\infty} \gamma_{n}<\infty$,

(iii) $\lim \sup _{n \rightarrow \infty} \delta_{n} \leq 0$.

Then, $\lim _{n \rightarrow \infty} a_{n}=0$.

Lemma 7 ([26]). Assume that $F: C \times C \rightarrow \mathbb{R}$ satisfies (A1)-(A4). For $u \in \mathscr{H}$ and $r>0$, define a mapping $T_{r}^{F}: \mathscr{H} \rightarrow C$ as follows:

$$
T_{r}^{F} u=\left\{w \in C: F(w, v)+\frac{1}{r}\langle v-w, w-u\rangle \geq 0, \quad \forall v \in C\right\} .
$$

Then, the following hold:

(i) $T_{r}^{F}$ is single-valued;

(ii) $T_{r}^{F}$ is a firmly nonexpansive mapping, i.e., for all $u, v \in H,\left\|T_{r}^{F} u-T_{r}^{F} v\right\|^{2} \leq\left\langle T_{r}^{F} u-\right.$ $\left.T_{r}^{F} v, u-v\right\rangle$;

(iii) $\operatorname{Fix}\left(T_{r}^{F}\right)=\mathrm{EP}(F)$;

(iv) $\mathrm{EP}(F)$ is closed and convex.

\section{Main Results}

Let $C$ and $Q$ be nonempty convex closed subsets of real Hilbert spaces $\mathscr{H}_{1}$ and $\mathscr{H}_{2}$, respectively, and $A: \mathscr{H}_{1} \rightarrow \mathscr{H}_{2}$ be a bounded linear operator with adjoint $A^{*}: \mathscr{H}_{2} \rightarrow \mathscr{H}_{1}$. Assume that $F_{1}: C \times C \rightarrow \mathbb{R}$ and $F_{2}: Q \times Q \rightarrow \mathbb{R}$ are bifunctions satisfying assumptions (A1)-(A4). Assume that $S: \mathscr{H}_{1} \rightarrow \mathscr{H}_{1}$ is $k$-demimetric and that $I-S$ is demiclosed at zero. Let $f: \mathscr{H}_{1} \rightarrow \mathscr{H}_{1}$ be contractive with constant $\alpha \in(0,1)$. Assume that Sol $:=$ $\operatorname{SEP}(F 1, F 2) \cap \operatorname{Fix}(S) \neq \varnothing$ and that the following conditions are satisfied:

(C1) $\left\{\alpha_{n}\right\} \subset(0,1), \lim _{n \rightarrow \infty} \alpha_{n}=0$ and $\sum_{n=1}^{\infty} \alpha_{n}=\infty$;

(C2) $\left\{\beta_{n}\right\} \subset(0,1), 0<\liminf _{n \rightarrow \infty} \beta_{n} \leq \limsup _{n \rightarrow \infty} \beta_{n}<1-k$;

(C3) $\left\{\mu_{n}\right\} \subset[0,1]$ and $\lim _{n \rightarrow \infty} \frac{\mu_{n}}{\alpha_{n}}\left\|x_{n}-x_{n-1}\right\|=0$, where $\left\{x_{n}\right\}$ is generated by Algorithm 3;

(C4) $\left\{r_{n}\right\} \subset(0, \infty)$ and $\liminf _{n \rightarrow \infty} r_{n}>0$.

We now introduce the following algorithm:

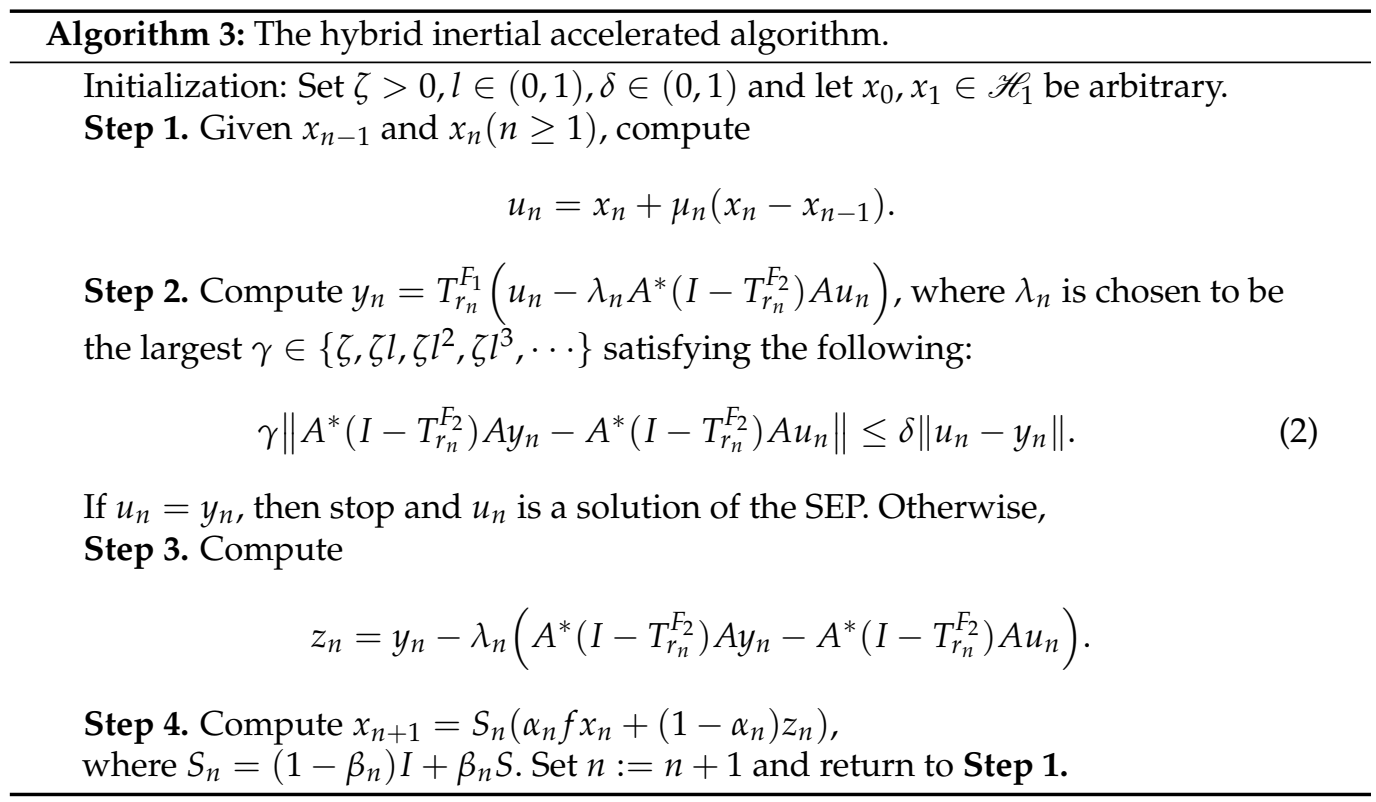

Before we prove the theorem, we need the following lemmas. 
Lemma 8. Suppose that $F: C \times C \rightarrow \mathbb{R}$ satisfies (A1)-(A4). For all $x \in \mathscr{H}$ and $0<a \leq b$, the following inequality holds:

$$
\frac{a}{b}\left\|x-T_{b}^{F} x\right\| \leq\left\|x-T_{a}^{F} x\right\| \leq\left\|x-T_{b}^{F} x\right\|
$$

Proof. Letting $x_{a}=T_{a}^{F} x$ and $x_{b}=T_{b}^{F} x$, we obtain

$$
F\left(x_{a}, y\right)+\frac{1}{a}\left\langle y-x_{a}, x_{a}-x\right\rangle \geq 0, \quad \forall y \in C
$$

and

$$
F\left(x_{b}, y\right)+\frac{1}{b}\left\langle y-x_{b}, x_{b}-x\right\rangle \geq 0, \quad \forall y \in C
$$

Hence,

$$
F\left(x_{a}, x_{b}\right)+\frac{1}{a}\left\langle x_{b}-x_{a}, x_{a}-x\right\rangle \geq 0
$$

and

$$
F\left(x_{b}, x_{a}\right)+\frac{1}{b}\left\langle x_{a}-x_{b}, x_{b}-x\right\rangle \geq 0 .
$$

Adding up (3) and (4) yields

$$
F\left(x_{a}, x_{b}\right)+F\left(x_{b}, x_{a}\right)+\left\langle x_{a}-x_{b}, \frac{x-x_{a}}{a}-\frac{x-x_{b}}{b}\right\rangle \geq 0 .
$$

Noticing (A2), we have

$$
0 \leq\left\langle x_{a}-x_{b}, \frac{x-x_{a}}{a}-\frac{x-x_{b}}{b}\right\rangle=\left\langle\left(x-x_{b}\right)-\left(x-x_{a}\right), \frac{x-x_{a}}{a}-\frac{x-x_{b}}{b}\right\rangle .
$$

This implies that

$$
0 \leq-\left\|x-x_{a}\right\|^{2}-\frac{a}{b}\left\|x-x_{b}\right\|^{2}+\left(1+\frac{a}{b}\right)\left\|x-x_{a}\right\|\left\|x-x_{b}\right\| .
$$

Therefore, we have

$$
\left(\left\|x-x_{a}\right\|-\frac{a}{b}\left\|x-x_{b}\right\|\right)\left(\left\|x-x_{a}\right\|-\left\|x-x_{b}\right\|\right) \leq 0
$$

which implies

$$
\left\|x-x_{a}\right\| \geq \frac{a}{b}\left\|x-x_{b}\right\| \text { and }\left\|x-x_{a}\right\| \leq\left\|x-x_{b}\right\| .
$$

Hence, we have

$$
\frac{a}{b}\left\|x-T_{b}^{F} x\right\| \leq\left\|x-T_{a}^{F} x\right\| \leq\left\|x-T_{b}^{F} x\right\|
$$

Lemma 9. Assume that $\mathscr{H}_{1}$ and $\mathscr{H}_{2}$ are real Hilbert spaces, $A: \mathscr{H}_{1} \rightarrow \mathscr{H}_{2}$ is a linear operator with its adjoint $A^{*}$. For any $r>0$ and $x \in \mathscr{H}_{1}$, define a mapping $\tilde{T}: \mathscr{H}_{1} \rightarrow \mathscr{H}_{1}$ as $\tilde{T}:=A^{*}\left(I-T_{r}^{F}\right) A$. Then, the following statements hold:

(1) $\quad\|\tilde{T} x-\tilde{T} y\| \leq\|A\|^{2}\|x-y\|$;

(2) $\left\|\left(I-T_{r}^{F}\right) A x-\left(I-T_{r}^{F}\right) A y\right\|^{2} \leq\langle\tilde{T} x-\tilde{T} y, x-y\rangle$. 
Proof. Since $T_{r}^{F}$ is a firmly nonexpansive mapping (Lemma 7(ii)), we deduce that $I-T_{r}^{F}$ is also firmly nonexpansive. Hence, we have that

$$
\begin{aligned}
\|\tilde{T} x-\tilde{T} y\| & \leq\|A\|\left\|\left(I-T_{r}^{F}\right) A x-\left(I-T_{r}^{F}\right) A y\right\| \\
& \leq\|A\|\|A x-A y\| \\
& \leq\|A\|^{2}\|x-y\|
\end{aligned}
$$

and

$$
\begin{aligned}
\left\|\left(I-T_{r}^{F}\right) A x-\left(I-T_{r}^{F}\right) A y\right\|^{2} & \leq\left\langle A x-A y,\left(I-T_{r}^{F}\right) A x-\left(I-T_{r}^{F}\right) A y\right\rangle \\
& =\left\langle x-y, A^{*}\left(I-T_{r}^{F}\right) A x-A^{*}\left(I-T_{r}^{F}\right) A y\right\rangle \\
& =\langle x-y, \tilde{T} x-\tilde{T} y\rangle . \quad \square
\end{aligned}
$$

Lemma 10. Assume that $\mathscr{H}_{1}$ and $\mathscr{H}_{2}$ are real Hilbert spaces, $A: \mathscr{H}_{1} \rightarrow \mathscr{H}_{2}$ is a linear operator with its adjoint $A^{*}$. Suppose that $\operatorname{SEP}(F 1, F 2) \neq \varnothing$ and $r>0$. Then, the following statements hold:

(1) If $\hat{x}$ is a solution of the SEP, then $T_{r}^{F_{1}}\left(\hat{x}-\lambda A^{*}\left(I-T_{r}^{F_{2}}\right) A \hat{x}\right)=\hat{x}$;

(2) Assume that $T_{r}^{F_{1}}\left(\hat{x}-\lambda A^{*}\left(I-T_{r}^{F_{2}}\right) A \hat{x}\right)=\hat{x}$. Then, $\hat{x}$ is a solution of the SEP.

Proof. (1) Assume that $\hat{x} \in \mathscr{H}_{1}$ is a solution of the SEP. Then, $\hat{x} \in \operatorname{EP}\left(F_{1}\right)$ and $A \hat{x} \in \operatorname{EP}\left(F_{2}\right)$. By Lemma 7(iii), it is easy to see that

$$
T_{r}^{F_{1}}\left(\hat{x}-\lambda A^{*}\left(I-T_{r}^{F_{2}}\right) A \hat{x}\right)=T_{r}^{F_{1}}(\hat{x})=\hat{x} .
$$

(2) Suppose that the solution set of the SEP is nonempty and that $T_{r}^{F_{1}}\left(\hat{x}-\lambda A^{*}\left(I-T_{r}^{F_{2}}\right) A \hat{x}\right)=$ $\hat{x}$. Then by Remark 1 , we have

$$
\left\langle\left(\hat{x}-\lambda A^{*}\left(I-T_{r}^{F_{2}}\right) A \hat{x}\right)-\hat{x}, z-\hat{x}\right\rangle \leq 0, \quad \forall z \in \operatorname{EP}\left(F_{1}\right) .
$$

That is,

$$
\left\langle A^{*}\left(I-T_{r}^{F_{2}}\right) A \hat{x}, \hat{x}-z\right\rangle \leq 0, \quad \forall z \in \operatorname{EP}\left(F_{1}\right) .
$$

Thus, we obtain

$$
\left\langle A \hat{x}-T_{r}^{F_{2}} A \hat{x}, A \hat{x}-A z\right\rangle \leq 0, \quad \forall z \in \operatorname{EP}\left(F_{1}\right) .
$$

On the other hand, by Remark 1 again,

$$
\left\langle A \hat{x}-T_{r}^{F_{2}} A \hat{x}, p-T_{r}^{F_{2}} A \hat{x}\right\rangle \leq 0, \quad \forall p \in \operatorname{EP}\left(F_{2}\right) .
$$

Adding (5) and (6), we get

$$
\left\langle A \hat{x}-T_{r}^{F_{2}} A \hat{x}, p-T_{r}^{F_{2}} A \hat{x}+A \hat{x}-A z\right\rangle \leq 0, \quad \forall z \in \operatorname{EP}\left(F_{1}\right), p \in \operatorname{EP}\left(F_{2}\right) .
$$

This implies

$$
\left\|A \hat{x}-T_{r}^{F_{2}} A \hat{x}\right\|^{2} \leq\left\langle A \hat{x}-T_{r}^{F_{2}} A \hat{x}, A z-p\right\rangle, \quad \forall z \in \operatorname{EP}\left(F_{1}\right), p \in \operatorname{EP}\left(F_{2}\right) .
$$

Suppose that $\bar{z} \in \mathscr{H}_{1}$ is a solution of the SEP. Then, $\bar{z} \in \operatorname{EP}\left(F_{1}\right)$ and $A \bar{z} \in \operatorname{EP}\left(F_{2}\right)$. Putting $z=\bar{z}$ and $p=A \bar{z}$ in (7), we deduce $A \hat{x} \in \operatorname{Fix}\left(T_{r}^{F_{2}}\right)=\operatorname{EP}\left(F_{2}\right)$. Furthermore,

$$
\hat{x}=T_{r}^{F_{1}}\left(\hat{x}-\lambda A^{*}\left(I-T_{r}^{F_{2}}\right) A \hat{x}\right)=T_{r}^{F_{1}} \hat{x} .
$$


Hence, $\hat{x} \in \operatorname{Fix}\left(T_{r}^{F_{1}}\right)=\operatorname{EP}\left(F_{1}\right)$. Therefore, $\hat{x}$ is a solution of the SEP.

Remark 1. By Lemma 7(ii), one can deduce (as in Remark 2.11 from Jolaoso and Karahan [13]) that, for any $u \in \mathscr{H}:$

$$
\left\langle T_{r}^{F} u-p, T_{r}^{F} u-u\right\rangle \leq 0, \quad \forall p \in \operatorname{Fix}\left(T_{r}^{F}\right) .
$$

Lemma 11. The Armijo-like search rule (2) is well-defined and $\min \left\{\zeta, \delta l\|A\|^{-2}\right\} \leq \lambda_{n} \leq \zeta$.

Proof. Indeed, using Lemma 9(1), one sees that

$$
\left\|A^{*}\left(I-T_{r_{n}}^{F_{2}}\right) A y_{n}-A^{*}\left(I-T_{r_{n}}^{F_{2}}\right) A u_{n}\right\| \leq\|A\|^{2}\left\|y_{n}-u_{n}\right\| .
$$

Obviously, (2) holds for all $0<\lambda_{n} \leq \delta\|A\|^{-2}$. On the other hand, it is easy to see that $\lambda_{n} \leq$ $\zeta$. If $\lambda_{n}=\zeta$; then, the lemma is proved. Otherwise, if $\lambda_{n}<\zeta$, then (2) will be violated when $\gamma=\lambda_{n} l^{-1}$, which indicates that $\lambda_{n} l^{-1}>\delta\|A\|^{-2}$. Hence, $\lambda_{n} \geq \min \left\{\zeta, \delta l\|A\|^{-2}\right\}$.

Lemma 12. Suppose that Conditions (C1)-(C4) hold. Let $\left\{u_{n}\right\},\left\{y_{n}\right\}$, and $\left\{z_{n}\right\}$ be three sequences created by Algorithm 3. Then, for $\forall q \in \operatorname{SEP}(F 1, F 2)$,

$$
\left\|z_{n}-q\right\|^{2} \leq\left\|u_{n}-q\right\|^{2}-\left(1-\delta^{2}\right)\left\|y_{n}-u_{n}\right\|^{2} .
$$

Proof. Let $q \in \operatorname{SEP}(F 1, F 2)$, that is $T_{r_{n}}^{F_{1}} q=q, T_{r_{n}}^{F_{2}} A q=A q$. From the definitions of $z_{n}$, we find

$$
\begin{aligned}
&\left\|z_{n}-q\right\|^{2} \\
&=\left\|y_{n}-\lambda_{n}\left(A^{*}\left(I-T_{r_{n}}^{F_{2}}\right) A y_{n}-A^{*}\left(I-T_{r_{n}}^{F_{2}}\right) A u_{n}\right)-q\right\|^{2} \\
&=\left\|y_{n}-q\right\|^{2}+\lambda_{n}^{2}\left\|A^{*}\left(I-T_{r_{n}}^{F_{2}}\right) A y_{n}-A^{*}\left(I-T_{r_{n}}^{F_{2}}\right) A u_{n}\right\|^{2} \\
&-2 \lambda_{n}\left\langle y_{n}-q, A^{*}\left(I-T_{r_{n}}^{F_{2}}\right) A y_{n}-A^{*}\left(I-T_{r_{n}}^{F_{2}}\right) A u_{n}\right\rangle \\
&=\left\|y_{n}-u_{n}\right\|^{2}+\left\|u_{n}-q\right\|^{2}+2\left\langle y_{n}-u_{n}, u_{n}-q\right\rangle+\lambda_{n}^{2}\left\|A^{*}\left(I-T_{r_{n}}^{F_{2}}\right) A y_{n}-A^{*}\left(I-T_{r_{n}}^{F_{2}}\right) A u_{n}\right\|^{2} \\
&-2 \lambda_{n}\left\langle y_{n}-q, A^{*}\left(I-T_{r_{n}}^{F_{2}}\right) A y_{n}-A^{*}\left(I-T_{r_{n}} y^{F}\right) A u_{n}\right\rangle \\
&=\left\|y_{n}-u_{n}\right\|^{2}+\left\|u_{n}-q\right\|^{2}-2\left\langle y_{n}-u_{n}, y_{n}-u_{n}\right\rangle+2\left\langle y_{n}-u_{n}, y_{n}-q\right\rangle \\
&+\lambda_{n}^{2}\left\|A^{*}\left(I-T_{r_{n}}^{F_{2}}\right) A y_{n}-A^{*}\left(I-T_{r_{n}}^{F_{2}}\right) A u_{n}\right\|^{2}-2 \lambda_{n}\left\langle y_{n}-q, A^{*}\left(I-T_{r_{n}}^{F_{2}}\right) A y_{n}-A^{*}\left(I-T_{r_{n}}^{F_{2}}\right) A u_{n}\right\rangle \\
& \quad\left\|u_{n}-q\right\|^{2}-\left\|y_{n}-u_{n}\right\|^{2}+2\left\langle y_{n}-u_{n}+\lambda_{n} A^{*}\left(I-T_{r_{n}}^{F_{2}}\right) A u_{n}, y_{n}-q\right\rangle \\
& \quad-2 \lambda_{n}\left\langle A^{*}\left(I-T_{r_{n}}^{F_{2}}\right) A y_{n}, y_{n}-q\right\rangle+\lambda_{n}^{2}\left\|A^{*}\left(I-T_{r_{n}}^{F_{2}}\right) A y_{n}-A^{*}\left(I-T_{r_{n}}^{F_{2}}\right) A u_{n}\right\|^{2} .
\end{aligned}
$$

Apply Remark 1 to obtain

$$
\left\langle y_{n}-u_{n}+\lambda_{n} A^{*}\left(I-T_{r_{n}}^{F_{2}}\right) A u_{n}, y_{n}-q\right\rangle \leq 0 .
$$

Noticing $A q=T_{r_{n}}^{F_{2}} A q$, we obtain from Lemma 9(2) that

$$
\left\langle A^{*}\left(I-T_{r_{n}}^{F_{2}}\right) A y_{n}, y_{n}-q\right\rangle \geq 0 .
$$


It follows from (2), (8), (9), and (10) that

$$
\begin{aligned}
\left\|z_{n}-q\right\|^{2} & \leq\left\|u_{n}-q\right\|^{2}-\left\|y_{n}-u_{n}\right\|^{2}+\lambda_{n}^{2}\left\|A^{*}\left(I-T_{r_{n}}^{F_{2}}\right) A y_{n}-A^{*}\left(I-T_{r_{n}}^{F_{2}}\right) A u_{n}\right\|^{2} \\
& \leq\left\|u_{n}-q\right\|^{2}-\left\|y_{n}-u_{n}\right\|^{2}+\delta^{2}\left\|y_{n}-u_{n}\right\|^{2} \\
& =\left\|u_{n}-q\right\|^{2}-\left(1-\delta^{2}\right)\left\|y_{n}-u_{n}\right\|^{2} .
\end{aligned}
$$

The proof is completed.

Lemma 13. Suppose that the sequences $\left\{u_{n}\right\}$ and $\left\{y_{n}\right\}$ are created by Algorithm 3. If $u_{n_{k}} \rightarrow z^{*}$ and $\lim _{n \rightarrow \infty}\left\|u_{n}-y_{n}\right\|=0$, then $z^{*} \in \operatorname{SEP}(F 1, F 2)$.

Proof. Taking any $q \in \operatorname{SEP}(F 1, F 2)$, we know that $T_{r_{n}}^{F_{2}} A q=A q$. This implies that $A^{*}(I-$ $\left.T_{r_{n}}^{F_{2}}\right) A q=0$. By Lemma 9(2), we obtain that

$$
\left\langle A^{*}\left(I-T_{r_{n}}^{F_{2}}\right) A y_{n}-A^{*}\left(I-T_{r_{n}}^{F_{2}}\right) A q, y_{n}-q\right\rangle \geq\left\|\left(I-T_{r_{n}}^{F_{2}}\right) A y_{n}\right\|^{2} .
$$

This together with (9) and Lemma 9(1) implies

$$
\begin{aligned}
\lambda_{n}\left\|A y_{n}-T_{r_{n}}^{F_{2}} A y_{n}\right\|^{2} \leq & \lambda_{n}\left\langle A^{*}\left(I-T_{r_{n}}^{F_{2}}\right) A y_{n}-A^{*}\left(I-T_{r_{n}}^{F_{2}}\right) A q, y_{n}-q\right\rangle \\
\leq & \lambda_{n}\left\langle A^{*}\left(I-T_{r_{n}}^{F_{2}}\right) A y_{n}-A^{*}\left(I-T_{r_{n}}^{F_{2}}\right) A q, y_{n}-q\right\rangle \\
& -\left\langle y_{n}-\left(u_{n}-\lambda_{n} A^{*}\left(I-T_{r_{n}}^{F_{2}}\right) A u_{n}\right), y_{n}-q\right\rangle \\
= & \left\langle u_{n}-y_{n}-\lambda_{n} A^{*}\left(I-T_{r_{n}}^{F_{2}}\right) A u_{n}+\lambda_{n} A^{*}\left(I-T_{r_{n}}^{F_{2}}\right) A y_{n}, y_{n}-q\right\rangle \\
\leq & \left\|u_{n}-y_{n}-\lambda_{n} A^{*}\left(I-T_{r_{n}}^{F_{2}}\right) A u_{n}+\lambda_{n} A^{*}\left(I-T_{r_{n}}^{F_{2}}\right) A y_{n}\right\|\left\|y_{n}-q\right\| \\
\leq & \left(\left\|u_{n}-y_{n}\right\|+\lambda_{n}\left\|A^{*}\left(I-T_{r_{n}}^{F_{2}}\right) A u_{n}-A^{*}\left(I-T_{r_{n}}^{F_{2}}\right) A y_{n}\right\|\right)\left\|y_{n}-q\right\| \\
\leq & \left(1+\lambda_{n}\|A\|^{2}\right)\left\|u_{n}-y_{n}\right\|\left\|y_{n}-q\right\| .
\end{aligned}
$$

Since $\min \left\{\zeta, \delta l\|A\|^{-2}\right\} \leq \lambda_{n} \leq \zeta$ and $\lim _{n \rightarrow \infty}\left\|u_{n}-y_{n}\right\|=0$, we find that $\lim _{n \rightarrow \infty}\left\|A y_{n}-T_{r_{n}}^{F_{2}} A y_{n}\right\|=0$. Moreover, by Lemma 7(ii), one can deduce

$$
\begin{aligned}
\left\|A u_{n}-T_{r_{n}}^{F_{2}} A u_{n}\right\| & \leq\left\|A u_{n}-A y_{n}+\left(T_{r_{n}}^{F_{2}} A y_{n}-T_{r_{n}}^{F_{2}} A u_{n}\right)\right\|+\left\|A y_{n}-T_{r_{n}}^{F_{2}} A y_{n}\right\| \\
& \leq 2\|A\|\left\|u_{n}-y_{n}\right\|+\left\|A y_{n}-T_{r_{n}}^{F_{2}} A y_{n}\right\| .
\end{aligned}
$$

This indicates that

$$
\lim _{n \rightarrow \infty}\left\|A u_{n}-T_{r_{n}}^{F_{2}} A u_{n}\right\|=0
$$

Again using Lemma 7(ii) and the definition of $y_{n}$, we derive

$$
\begin{aligned}
\left\|y_{n}-T_{r_{n}}^{F_{1}} u_{n}\right\| & =\left\|T_{r_{n}}^{F_{1}}\left(u_{n}-\lambda_{n} A^{*}\left(I-T_{r_{n}}^{F_{2}}\right) A u_{n}\right)-T_{r_{n}}^{F_{1}} u_{n}\right\| \\
& \leq\left\|\lambda_{n} A^{*}\left(I-T_{r_{n}}^{F_{2}}\right) A u_{n}\right\| \\
& \leq \lambda_{n}\|A\|\left\|A u_{n}-T_{r_{n}}^{F_{2}} A u_{n}\right\|,
\end{aligned}
$$

which together with (11) gives that $\lim _{n \rightarrow \infty}\left\|y_{n}-T_{r_{n}}^{F_{1}} u_{n}\right\|=0$. From $\lim _{n \rightarrow \infty}\left\|y_{n}-u_{n}\right\|=0$, one obtains $\lim _{n \rightarrow \infty}\left\|u_{n}-T_{r_{n}}^{F_{1}} u_{n}\right\|=0$. 
According to (C4), there exists a positive number $r$ and some positive integer $N_{0}$ such that $0<r<r_{n}\left(\forall n \geq N_{0}\right)$. It follows from Lemma 8 that

$$
\lim _{n \rightarrow \infty}\left\|u_{n}-T_{r}^{F_{1}} u_{n}\right\| \leq \lim _{n \rightarrow \infty}\left\|u_{n}-T_{r_{n}}^{F_{1}} u_{n}\right\|=0
$$

This combining with Lemma 3, Lemma 7(ii), and $u_{n_{k}} \rightarrow z^{*}$ yields $z^{*} \in \operatorname{Fix}\left(T_{r}^{F_{1}}\right)=\operatorname{EP}\left(F_{1}\right)$. Due to the fact that $A$ is a linear bounded operator and $u_{n_{k}} \rightarrow z^{*}$, we get

$$
A u_{n_{k}} \rightarrow A z^{*} .
$$

Using (11) and Lemma 8, we obtain

$$
\lim _{n \rightarrow \infty}\left\|A u_{n}-T_{r}^{F_{2}} A u_{n}\right\| \leq \lim _{n \rightarrow \infty}\left\|A u_{n}-T_{r_{n}}^{F_{2}} A u_{n}\right\|=0 .
$$

By (12), (13), Lemma 3, and Lemma 7(iii), we get $A z^{*} \in \operatorname{Fix}\left(T_{r}^{F_{2}}\right)=\operatorname{EP}\left(F_{2}\right)$. Thus, we deduce that $z^{*} \in \operatorname{SEP}(F 1, F 2)$. The proof is completed.

Theorem 2. Assume that conditions (C1)-(C4) are satisfied. Then, the iterative sequence $\left\{x_{n}\right\}$ constructed by Algorithm 3 converges to $q$ in the norm, where $q=P_{\text {Sol }} f q$.

Remark 2. We note that the condition (C3) can be easily implemented due to the fact that the value of $\left\|x_{n}-x_{n-1}\right\|$ is known before choosing $\mu_{n}$. Indeed, the parameter $\mu_{n}$ can be chosen such that

$$
\mu_{n}= \begin{cases}\omega, & x_{n}=x_{n-1}, \\ \frac{\xi_{n}}{\left\|x_{n}-x_{n-1}\right\|}, & x_{n} \neq x_{n-1},\end{cases}
$$

where $\omega \geq 0$ and $\left\{\xi_{n}\right\}$ is a positive sequence such that $\xi_{n}=o\left(\alpha_{n}\right)$.

We now prove the Theorem 2.

Proof. First, we prove that the sequence $\left\{x_{n}\right\}$ is bounded. Taking any $q \in$ Sol, we infer from Lemma 12 that

$$
\left\|z_{n}-q\right\| \leq\left\|u_{n}-q\right\|
$$

In view of the definition of $u_{n}$, one deduces

$$
\left\|u_{n}-q\right\| \leq\left\|x_{n}-q\right\|+\mu_{n}\left\|x_{n}-x_{n-1}\right\| .
$$

Invoking (C3), there exists a positive constant $M_{1}<\infty$ such that

$$
\frac{\mu_{n}}{\alpha_{n}}\left\|x_{n}-x_{n-1}\right\| \leq M_{1} .
$$


From (14), (15), and Lemma 4, one obtains

$$
\begin{aligned}
\left\|x_{n+1}-q\right\| & =\left\|S_{n}\left(\alpha_{n} f x_{n}+\left(1-\alpha_{n}\right) z_{n}\right)-q\right\| \\
& \leq\left\|\alpha_{n}\left(f x_{n}-q\right)+\left(1-\alpha_{n}\right)\left(z_{n}-q\right)\right\| \\
& \leq \alpha_{n}\left\|f x_{n}-f q\right\|+\alpha_{n}\|f q-q\|+\left(1-\alpha_{n}\right)\left\|z_{n}-q\right\| \\
& \leq \alpha_{n} \alpha\left\|x_{n}-q\right\|+\alpha_{n}\|f q-q\|+\left(1-\alpha_{n}\right)\left(\left\|x_{n}-q\right\|+\mu_{n}\left\|x_{n}-x_{n-1}\right\|\right) \\
& \leq\left(1-(1-\alpha) \alpha_{n}\right)\left\|x_{n}-q\right\|+\alpha_{n}\|f q-q\|+\alpha_{n} M_{1} \\
& \leq\left(1-(1-\alpha) \alpha_{n}\right)\left\|x_{n}-q\right\|+\alpha_{n}(1-\alpha) \frac{\|f q-q\|+M_{1}}{1-\alpha} \\
& \leq \max \left\{\left\|x_{n}-q\right\|, \frac{\|f q-q\|+M_{1}}{1-\alpha}\right\} \\
& \leq \cdots \leq \max \left\{\left\|x_{1}-q\right\|, \frac{\|f q-q\|+M_{1}}{1-\alpha}\right\} .
\end{aligned}
$$

This implies that sequence $\left\{x_{n}\right\}$ is bounded. At the same time, using (14), (15), and the definition of $\left\{y_{n}\right\}$, one concludes that $\left\{z_{n}\right\},\left\{y_{n}\right\}$, and $\left\{u_{n}\right\}$ are bounded. According to Lemma 1(3), we obtain

$$
\begin{aligned}
\left\|u_{n}-q\right\|^{2} & =\left\|x_{n}-q+\mu_{n}\left(x_{n}-x_{n-1}\right)\right\|^{2} \\
& \leq\left\|x_{n}-q\right\|^{2}+2 \mu_{n}\left\langle x_{n}-x_{n-1}, u_{n}-q\right\rangle \\
& \leq\left\|x_{n}-q\right\|^{2}+2 \mu_{n}\left\|x_{n-1}-x_{n}\right\|\left\|u_{n}-q\right\| \\
& \leq\left\|x_{n}-q\right\|^{2}+\mu_{n}\left\|x_{n-1}-x_{n}\right\| M_{2},
\end{aligned}
$$

where $M_{2}=\sup _{n>0}\left\{2\left\|u_{n}-q\right\|\right\}<\infty$.

It follows from Lemma 4, Lemma 12, and (16) that

$$
\begin{aligned}
& \left\|x_{n+1}-q\right\|^{2} \\
= & \left\|S_{n}\left(\alpha_{n} f x_{n}+\left(1-\alpha_{n}\right) z_{n}\right)-q\right\|^{2} \\
\leq & \left\|\alpha_{n} f x_{n}+\left(1-\alpha_{n}\right) z_{n}-q\right\|^{2} \\
\leq & \alpha_{n}\left\|f x_{n}-q\right\|^{2}+\left(1-\alpha_{n}\right)\left\|z_{n}-q\right\|^{2} \\
\leq & \alpha_{n}\left\|f x_{n}-q\right\|^{2}+\left\|u_{n}-q\right\|^{2}-\left(1-\delta^{2}\right)\left\|y_{n}-u_{n}\right\|^{2}, \\
\leq & \alpha_{n}\left\|f x_{n}-q\right\|^{2}+\left\|x_{n}-q\right\|^{2}+\mu_{n}\left\|x_{n-1}-x_{n}\right\| M_{2}-\left(1-\delta^{2}\right)\left\|y_{n}-u_{n}\right\|^{2}, \\
\leq & \alpha_{n} M_{3}+\left\|x_{n}-q\right\|^{2}-\left(1-\delta^{2}\right)\left\|y_{n}-u_{n}\right\|^{2},
\end{aligned}
$$

where $M_{3}=\sup _{n \geq 1}\left\{\left\|f x_{n}-q\right\|^{2}+\frac{\mu_{n}}{\alpha_{n}}\left\|x_{n-1}-x_{n}\right\| M_{2}\right\}<\infty$. Let us rewrite (17) as

$$
\left(1-\delta^{2}\right)\left\|y_{n}-u_{n}\right\|^{2} \leq \alpha_{n} M_{3}+\left\|x_{n}-q\right\|^{2}-\left\|x_{n+1}-q\right\|^{2} .
$$

Setting $g_{n}=\alpha_{n} f x_{n}+\left(1-\alpha_{n}\right) z_{n}$ and using (14), (16), and Lemma 1(1), we infer

$$
\begin{aligned}
& \left\|x_{n+1}-q\right\|^{2} \\
= & \left\|\left(\left(1-\beta_{n}\right) I+\beta_{n} S\right) g_{n}-q\right\|^{2} \\
= & \left(1-\beta_{n}\right)\left\|g_{n}-q\right\|^{2}+\beta_{n}\left\|S g_{n}-q\right\|^{2}-\beta_{n}\left(1-\beta_{n}\right)\left\|g_{n}-S g_{n}\right\|^{2} \\
\leq & \left(\left(1-\beta_{n}\right)\left\|g_{n}-q\right\|^{2}+\beta_{n}\left(\left\|g_{n}-q\right\|^{2}+k\left\|g_{n}-S g_{n}\right\|^{2}\right)-\beta_{n}\left(1-\beta_{n}\right)\left\|g_{n}-S g_{n}\right\|^{2}\right. \\
= & \left\|g_{n}-q\right\|^{2}-\beta_{n}\left(1-\beta_{n}-k\right)\left\|g_{n}-S g_{n}\right\|^{2} \\
\leq & \alpha_{n}\left\|f x_{n}-q\right\|^{2}+\left(1-\alpha_{n}\right)\left\|z_{n}-q\right\|^{2}-\beta_{n}\left(1-\beta_{n}-k\right)\left\|g_{n}-S g_{n}\right\|^{2} \\
\leq & \alpha_{n}\left\|f x_{n}-q\right\|^{2}+\left\|x_{n}-q\right\|^{2}+\mu_{n}\left\|x_{n-1}-x_{n}\right\| M_{2}-\beta_{n}\left(1-\beta_{n}-k\right)\left\|g_{n}-S g_{n}\right\|^{2} .
\end{aligned}
$$

Thus, 


$$
\beta_{n}\left(1-\beta_{n}-k\right)\left\|g_{n}-S g_{n}\right\|^{2} \leq\left\|x_{n}-q\right\|^{2}-\left\|x_{n+1}-q\right\|^{2}+\alpha_{n}\left\|f x_{n}-q\right\|^{2}+\mu_{n}\left\|x_{n-1}-x_{n}\right\| M_{2} \text {. }
$$

We next show the convergence of $\left\{\left\|x_{n}-q\right\|\right\}$ to zero by the following two cases:

Case 1. Assume that there exists $N_{0} \in \mathbb{N}$ such that the sequence $\left\{\left\|x_{n}-q\right\|\right\}_{n \geq N_{0}}$ is monotonously decreasing; then, $\lim _{n \rightarrow \infty}\left\|x_{n}-q\right\|$ exists. From (C1) and putting $n$ tending to infinity in (18), we derive that

$$
\lim _{n \rightarrow \infty}\left\|y_{n}-u_{n}\right\|=0 .
$$

It follows from (C1), (C3), and the definitions of $u_{n}$ that

$$
\lim _{n \rightarrow \infty}\left\|u_{n}-x_{n}\right\|=\lim _{n \rightarrow \infty} \mu_{n}\left\|x_{n}-x_{n-1}\right\|=0 .
$$

By (C1), (C2), (C3), and (19), we obtain

$$
\lim _{n \rightarrow \infty}\left\|g_{n}-S g_{n}\right\|=0
$$

Due to the fact that $g_{n}=\alpha_{n} f x_{n}+\left(1-\alpha_{n}\right) z_{n}$, we infer

$$
\lim _{n \rightarrow \infty}\left\|g_{n}-z_{n}\right\|=\lim _{n \rightarrow \infty} \alpha_{n}\left\|f x_{n}-z_{n}\right\|=0 .
$$

Noticing $\min \left\{\zeta, \delta l\|A\|^{-2}\right\} \leq \lambda_{n} \leq \zeta$ and resorting to the definition of $z_{n}$, Lemma 9(1), and (20), one deduces that

$\lim _{n \rightarrow \infty}\left\|z_{n}-y_{n}\right\|=\lambda_{n}\left\|A^{*}\left(I-T_{r_{n}}^{F_{2}}\right) A y_{n}-A^{*}\left(I-T_{r_{n}}^{F_{2}}\right) A u_{n}\right\| \leq \lim _{n \rightarrow \infty} \lambda_{n}\|A\|^{2}\left\|y_{n}-u_{n}\right\|=0$.

Thanks to (20), (21) and (24), one infers that

$$
\lim _{n \rightarrow \infty}\left\|z_{n}-x_{n}\right\|=0
$$

Taking into consideration that

$$
\begin{aligned}
\left\|x_{n+1}-z_{n}\right\| & =\left\|\left(\left(1-\beta_{n}\right) I+\beta_{n} S\right) g_{n}-z_{n}\right\| \\
& \leq\left(1-\beta_{n}\right)\left\|g_{n}-z_{n}\right\|+\beta_{n}\left(\left\|S g_{n}-g_{n}\right\|+\left\|g_{n}-z_{n}\right\|\right),
\end{aligned}
$$

we deduce from (22) and (23) that

$$
\lim _{n \rightarrow \infty}\left\|x_{n+1}-z_{n}\right\|=0
$$

Noticing $\left\|x_{n+1}-x_{n}\right\| \leq\left\|x_{n+1}-z_{n}\right\|+\left\|z_{n}-x_{n}\right\|$, this together with (25) and (26) implies

$$
\lim _{n \rightarrow \infty}\left\|x_{n+1}-x_{n}\right\|=0 \text {. }
$$

As $\left\{x_{n}\right\}$ is bounded, it indicates that there exists a subsequence $\left\{x_{n_{k}}\right\}$ of $\left\{x_{n}\right\}$ that converges weakly to some $z \in \mathscr{H}_{1}$ and

$$
\limsup _{n \rightarrow \infty}\left\langle f q-q, x_{n}-q\right\rangle=\lim _{k \rightarrow \infty}\left\langle f q-q, x_{n_{k}}-q\right\rangle=\langle f q-q, z-q\rangle
$$

According to (20), (21), and Lemma 13, we derive $z \in \operatorname{SEP}(F 1, F 2)$. By the assumption that $I-S$ is demiclosed and noticing that (22), (23), and (25), we deduce $z \in \operatorname{Fix}(S)$. Therefore, $z \in$ Sol. It is easy to see that $P_{\text {Sol }} f$ is a contractive mapping. Banach's Contraction Mapping 
Principle implies that $P_{\text {Sol }} f$ has a unique fixed point, say, $q \in \mathscr{H}_{1}$ and namely, $q=P_{\text {Sol }} f q$. It follows from Lemma 2 that

$$
\langle f q-q, y-q\rangle \leq 0, \quad \forall y \in \text { Sol. }
$$

Therefore, we have

$$
\limsup _{n \rightarrow \infty}\left\langle f q-q, x_{n}-q\right\rangle=\lim _{k \rightarrow \infty}\left\langle f q-q, x_{n_{k}}-q\right\rangle=\langle f q-q, z-q\rangle \leq 0 .
$$

This together with (27) implies that

$$
\limsup _{n \rightarrow \infty}\left\langle f q-q, x_{n+1}-q\right\rangle \leq 0 .
$$

It follows from (14), (16), Lemma 1(3), and Lemma 4 that

$$
\begin{aligned}
& \left\|x_{n+1}-q\right\|^{2} \\
\leq & \left\|\alpha_{n} f x_{n}+\left(1-\alpha_{n}\right) z_{n}-q\right\|^{2} \\
= & \left\|\alpha_{n}\left(f x_{n}-f q\right)+\left(1-\alpha_{n}\right)\left(z_{n}-q\right)+\alpha_{n}(f q-q)\right\|^{2} \\
\leq & \left\|\alpha_{n}\left(f x_{n}-f q\right)+\left(1-\alpha_{n}\right)\left(z_{n}-q\right)\right\|^{2}+2 \alpha_{n}\left\langle f q-q, x_{n+1}-q\right\rangle \\
\leq & \alpha_{n}\left\|f x_{n}-f q\right\|^{2}+\left(1-\alpha_{n}\right)\left\|z_{n}-q\right\|^{2}+2 \alpha_{n}\left\langle f q-q, x_{n+1}-q\right\rangle \\
\leq & \alpha_{n} \alpha\left\|x_{n}-q\right\|^{2}+\left(1-\alpha_{n}\right)\left(\left\|x_{n}-q\right\|^{2}+\mu_{n}\left\|x_{n}-x_{n-1}\right\| M_{2}\right)+2 \alpha_{n}\left\langle f q-q, x_{n+1}-q\right\rangle \\
\leq & \left(1-\alpha_{n}(1-\alpha)\right)\left\|x_{n}-q\right\|^{2}+\alpha_{n}\left(\frac{\mu_{n}}{\alpha_{n}}\left\|x_{n}-x_{n-1}\right\| M_{2}\right)+2 \alpha_{n}\left\langle f q-q, x_{n+1}-q\right\rangle .
\end{aligned}
$$

Thus, from (28), (C1), (C3), and Lemma 6, we conclude that $x_{n} \rightarrow q$.

Case 2. Assume that $\left\{\left\|x_{n}-q\right\|\right\}$ is not monotonously decreasing. Then, there exists a subsequence $\left\{\left\|x_{n_{i}}-q\right\|\right\}$ of $\left\{\left\|x_{n}-q\right\|\right\}$ such that

$$
\left\|x_{n_{i}}-q\right\|<\left\|x_{n_{i}+1}-q\right\|, \quad \forall i \in \mathbb{N} .
$$

According to Lemma 5, there exists a nondecreasing sequence $\left\{m_{k}\right\} \subset \mathbb{N}$ such that

$$
\max \left\{\left\|x_{m_{k}}-q\right\|,\left\|x_{k}-q\right\|\right\} \leq\left\|x_{m_{k}+1}-q\right\| .
$$

Following a similar argument as in Case I, it is easy to obtain

$$
\lim _{k \rightarrow \infty}\left\|x_{m_{k}+1}-x_{m_{k}}\right\|=0
$$

We want to show that

$$
\limsup _{k \rightarrow \infty}\left\langle f(q)-q, x_{m_{k}+1}-q\right\rangle \leq 0,
$$

where $q=P_{\text {Sol }} f q$. Without loss of generality, there exists a subsequence $\left\{x_{m_{k_{j}}}\right\}$ of $\left\{x_{m_{k}}\right\}$ such that $x_{m_{k_{j}}} \rightarrow w$ for some $w \in \mathscr{H}_{1}$ and

$$
\limsup _{k \rightarrow \infty}\left\langle f(q)-q, x_{m_{k}}-q\right\rangle=\lim _{j \rightarrow \infty}\left\langle f(q)-q, x_{m_{k_{j}}}-q\right\rangle .
$$

Like in Case 1, we can also obtain $\omega \in$ Sol. Thus, we have by Lemma 2 that

$$
\begin{aligned}
\limsup _{k \rightarrow \infty}\left\langle f(q)-q, x_{m_{k}}-q\right\rangle & =\lim _{j \rightarrow \infty}\left\langle f(q)-q, x_{m_{k_{j}}}-q\right\rangle \\
& =\left\langle f(q)-P_{\text {Sol }} f(q), w-P_{\text {Sol }} f(q)\right\rangle \\
& \leq 0 .
\end{aligned}
$$


This together with (31) implies

$$
\limsup _{k \rightarrow \infty}\left\langle f(q)-q, x_{m_{k}+1}-q\right\rangle \leq 0 .
$$

Resorting to (14), (16), Lemma 1(3), and Lemma 4, one deduces that

$$
\begin{aligned}
& \left\|x_{m_{k}+1}-q\right\|^{2} \\
= & \left\|S_{m_{k}}\left(\alpha_{m_{k}} f x_{m_{k}}+\left(1-\alpha_{m_{k}}\right) z_{m_{k}}\right)-q\right\|^{2} \\
\leq & \left\|\alpha_{m_{k}}\left(f x_{m_{k}}-f q\right)+\left(1-\alpha_{m_{k}}\right)\left(z_{m_{k}}-q\right)+\alpha_{m_{k}}(f q-q)\right\|^{2} \\
\leq & \left\|\alpha_{m_{k}}\left(f x_{m_{k}}-f q\right)+\left(1-\alpha_{m_{k}}\right)\left(z_{m_{k}}-q\right)\right\|^{2}+2 \alpha_{m_{k}}\left\langle f q-q, x_{m_{k}+1}-q\right\rangle \\
\leq & \alpha_{m_{k}}\left\|f x_{m_{k}}-f q\right\|^{2}+\left(1-\alpha_{m_{k}}\right)\left\|z_{m_{k}}-q\right\|^{2}+2 \alpha_{m_{k}}\left\langle f q-q, x_{m_{k}+1}-q\right\rangle \\
\leq & \alpha_{m_{k}} \alpha\left\|x_{m_{k}}-q\right\|^{2}+\left(1-\alpha_{m_{k}}\right)\left\|u_{m_{k}}-q\right\|^{2}+2 \alpha_{m_{k}}\left\langle f q-q, x_{m_{k}+1}-q\right\rangle \\
\leq & \alpha_{m_{k}} \alpha\left\|x_{m_{k}}-q\right\|^{2}+\left(1-\alpha_{m_{k}}\right)\left(\left\|x_{m_{k}}-q\right\|^{2}+\mu_{m_{k}}\left\|x_{m_{k}}-x_{m_{k}-1}\right\| M_{2}\right)+2 \alpha_{m_{k}}\left\langle f q-q, x_{m_{k}+1}-q\right\rangle \\
\leq & \left(1-\alpha_{m_{k}}(1-\alpha)\right)\left\|x_{m_{k}}-q\right\|^{2}+\alpha_{m_{k}}\left(\frac{\mu_{n}}{\alpha_{m_{k}}}\left\|x_{m_{k}}-x_{m_{k}-1}\right\| M_{2}\right)+2 \alpha_{m_{k}}\left\langle f q-q, x_{m_{k}+1}-q\right\rangle,
\end{aligned}
$$

which yields that

$$
\begin{aligned}
& (1-\alpha) \alpha_{m_{k}}\left\|x_{m_{k}+1}-q\right\|^{2} \\
\leq & \left(1-\alpha_{m_{k}}(1-\alpha)\right)\left(\left\|x_{m_{k}}-q\right\|^{2}-\left\|x_{m_{k}+1}-q\right\|^{2}\right)+\alpha_{m_{k}}\left(\frac{\mu_{n}}{\alpha_{m_{k}}}\left\|x_{m_{k}}-x_{n-1}\right\| M_{2}\right) \\
+ & 2 \alpha_{m_{k}}\left\langle f q-q, x_{m_{k}+1}-q\right\rangle .
\end{aligned}
$$

Noticing (29), we infer

$$
\left\|x_{m_{k}+1}-q\right\|^{2} \leq \frac{1}{1-\alpha}\left(\frac{\mu_{n}}{\alpha_{m_{k}}}\left\|x_{m_{k}}-x_{n-1}\right\| M_{2}\right)+\frac{2}{1-\alpha}\left\langle f q-q, x_{m_{k}+1}-q\right\rangle .
$$

By applying (C1), (C3), and (32), we get

$$
\lim _{k \rightarrow \infty}\left\|x_{m_{k}+1}-q\right\|=0 .
$$

It thus follows from (30) that

$$
\lim _{k \rightarrow \infty}\left\|x_{k}-q\right\|=0 .
$$

From the above, one can conclude that the sequences constructed by Algorithm 3 converge strongly to $q \in \mathrm{Sol}$, which is the unique fixed point of the contractive mapping $P_{\text {Sol }} f$. This completes the proof.

Remark 3. Compared with the known results in the literature, our results are very different from those in the following aspects.

- $\quad$ The proofs of our main results are simple and different from those in early and recent literature manly due to Lemma 8. More precisely, Lemma 8 together with Lemma 13 presents an interesting and simple method to prove $u_{n} \rightarrow p \in \operatorname{SEP}(F 1, F 2)$ under the conditions $u_{n} \rightarrow p$ and $\left\|u_{n}-T_{r_{n}}^{F} u_{n}\right\| \rightarrow 0$.

- $\quad$ Theorem 2 extends, improves, and develops the corresponding results in [12-14,17] from finding a solution for the VIP, a solution for the the EP, or a common solution for the SEP and the fixed point problem for demicontractive mappings to finding a common solution for the SEP and the fixed point problem for demimetric mappings. Moreover, our proof is also different from the one used in those paper.

- $\quad$ The numerical results have shown the effectiveness and fast convergence of our iterative scheme over the iterative schemes in [12-14,17]. 
Corollary 1. Assume that Conditions (C1)-(C4) are satisfied. Then, the sequence $\left\{x_{n}\right\}$ constructed by Algorithm 4 converges strongly to a point $q$, where $q=P_{\mathrm{SVIP}(C, Q)} f q$.

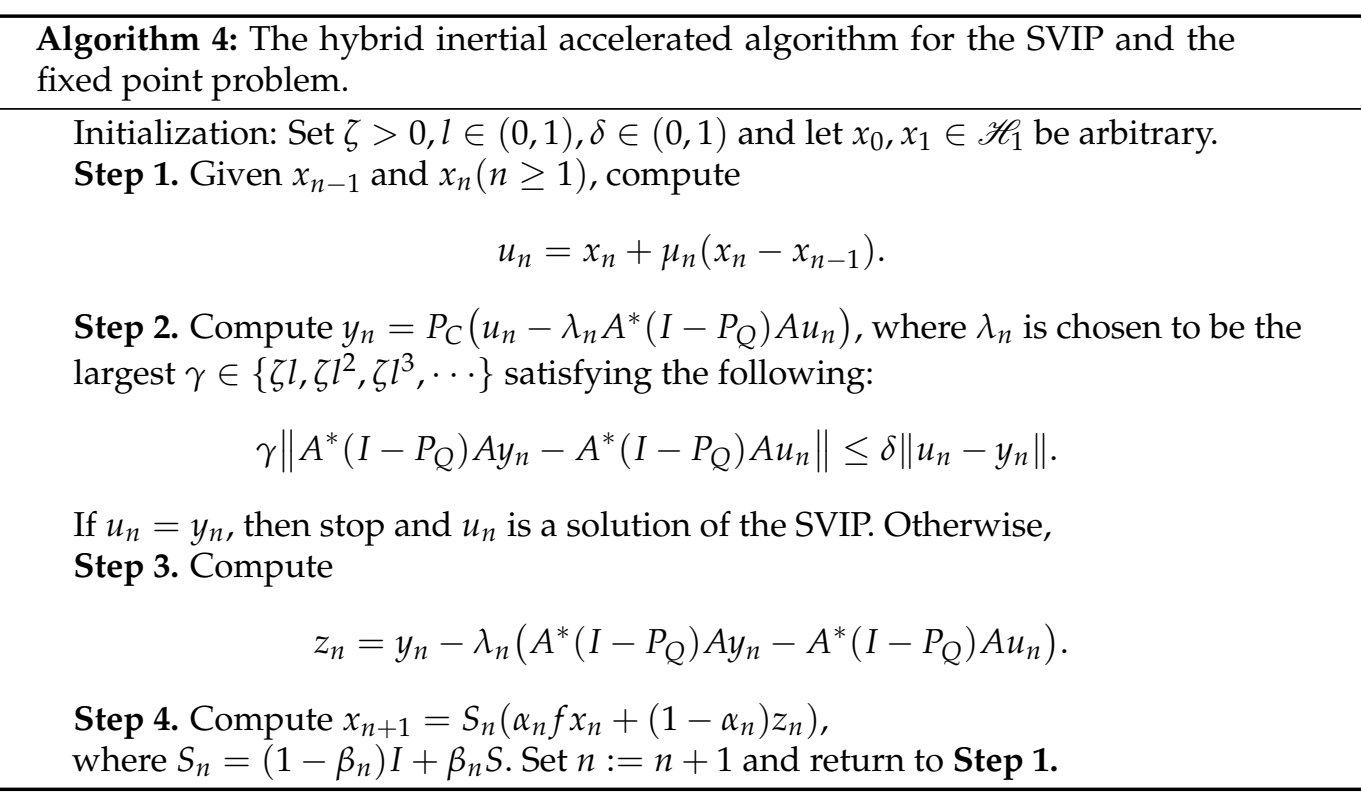

Proof. In Theorem 2, put $F_{1}(x, y)=0, \forall x, y \in C$ and $F_{2}(u, v)=0, \forall u, v \in Q$. Then we have that $T_{r_{n}}^{F_{1}}=P_{C}$ and $T_{r_{n}}^{F_{2}}=P_{\mathrm{Q}}$ for all $n \in \mathbb{N}$. Thus we obtain the desired result from Lemma 2 and Theorem 2.

\section{Application to Split Minimization Problems}

Let $C$ be a nonempty, closed, and convex subset of a Hilbert space $\mathscr{H}$. The constrained convex minimization problem is to find a point $v^{*} \in C$ such that

$$
\psi\left(v^{*}\right)=\min _{v \in C} \psi(v),
$$

where $\psi: C \rightarrow \mathbb{R}$ is a continuous differentiable function.

Lemma 14 ([15,27]). A necessary condition of optimality for a point $v^{*} \in C$ to be a solution of the minimization problem (33) is that $v^{*}$ solves the inequality

$$
\left\langle\nabla \psi\left(v^{*}\right), u-v^{*}\right\rangle \geq 0, \quad \forall u \in C .
$$

Equivalently, $v^{*} \in C$ solves the fixed point equation $v^{*}=P_{C}\left(v^{*}-\gamma \nabla \psi\left(v^{*}\right)\right)$, for every constant $\gamma>0$. If, in addition, $\psi$ is convex, then the optimality condition (34) is also sufficient.

Setting $F(z, v)=\langle\nabla \psi(z), v-z\rangle$, we can easily deduce that $F(z, v)$ satisfies condition (A1)-(A4) and $\operatorname{Argmin}(\psi)=\operatorname{EP}(F)$. Define a resolvent operator $T_{\gamma}^{\nabla \psi}: \mathscr{H} \rightarrow C$ as

$$
T_{\gamma}^{\nabla \psi} u=\left\{z \in C:\langle\nabla \psi(z), v-z\rangle+\frac{1}{\gamma}\langle v-z, z-u\rangle \geq 0, \quad \forall v \in C\right\} .
$$

From Lemma 7(iii), we get $\operatorname{Fix}\left(T_{\gamma}^{\nabla \psi}\right)=\operatorname{EP}(F)=\operatorname{Argmin}(\psi)$. Let us recall the split convex minimization problem (SCMP) as follows:

$$
\text { finding } v^{*} \in \operatorname{Argmin}\left(\psi_{1}\right) \text { such that } A v^{*} \in \operatorname{Argmin}\left(\psi_{2}\right) \text {, }
$$

where $C$ and $Q$ are nonempty convex closed subsets of real Hilbert spaces $\mathscr{H}_{1}$ and $\mathscr{H}_{2}$, respectively, $\psi_{1}: C \rightarrow \mathbb{R}$ and $\psi_{2}: Q \rightarrow \mathbb{R}$ are two proper convex differentiable functions, 
A $: \mathscr{H}_{1} \rightarrow \mathscr{H}_{2}$ is a bounded linear operator with adjoint $A^{*}: \mathscr{H}_{2} \rightarrow \mathscr{H}_{1}$. We denote the set of solutions of Problem (35) by $\operatorname{SCMP}\left(\psi_{1}, \psi_{2}\right)$. By setting $T_{r}^{F_{1}}=T_{r}^{\nabla \psi_{1}}$ and $T_{r}^{F_{2}}=T_{r}^{\nabla \psi_{2}}$, we have the following theorem follows directly from Theorem 2.

Theorem 3. Let $C$ and $Q$ be nonempty closed convex subsets of real Hilbert spaces $\mathscr{H}_{1}$ and $\mathscr{H}_{2}$, respectively. Assume that $A: \mathscr{H}_{1} \rightarrow \mathscr{H}_{2}$ is a bounded linear operator with adjoint $A^{*}: \mathscr{H}_{2} \rightarrow$ $\mathscr{H}_{1}, \psi_{1}: C \rightarrow \mathbb{R}$ and $\psi_{2}: Q \rightarrow \mathbb{R}$ are proper convex differentiable functions. Suppose that $S: \mathscr{H}_{1} \rightarrow \mathscr{H}_{1}$ is k-demimetric such that $I-S$ is demiclosed at zero and that $f: \mathscr{H}_{1} \rightarrow \mathscr{H}_{1}$ is $\alpha$-contractive with constant $\alpha \in(0,1)$. Suppose that the conditions (C1)-(C4) hold and Sol $:=$ $\operatorname{SCMP}\left(\psi_{1}, \psi_{2}\right) \cap \operatorname{Fix}(S) \neq \varnothing$. Then, the iterative sequence $\left\{x_{n}\right\}$ constructed by Algorithm 5 converges to $q$ in the norm, where $q=P_{\text {Sol }} f q$.

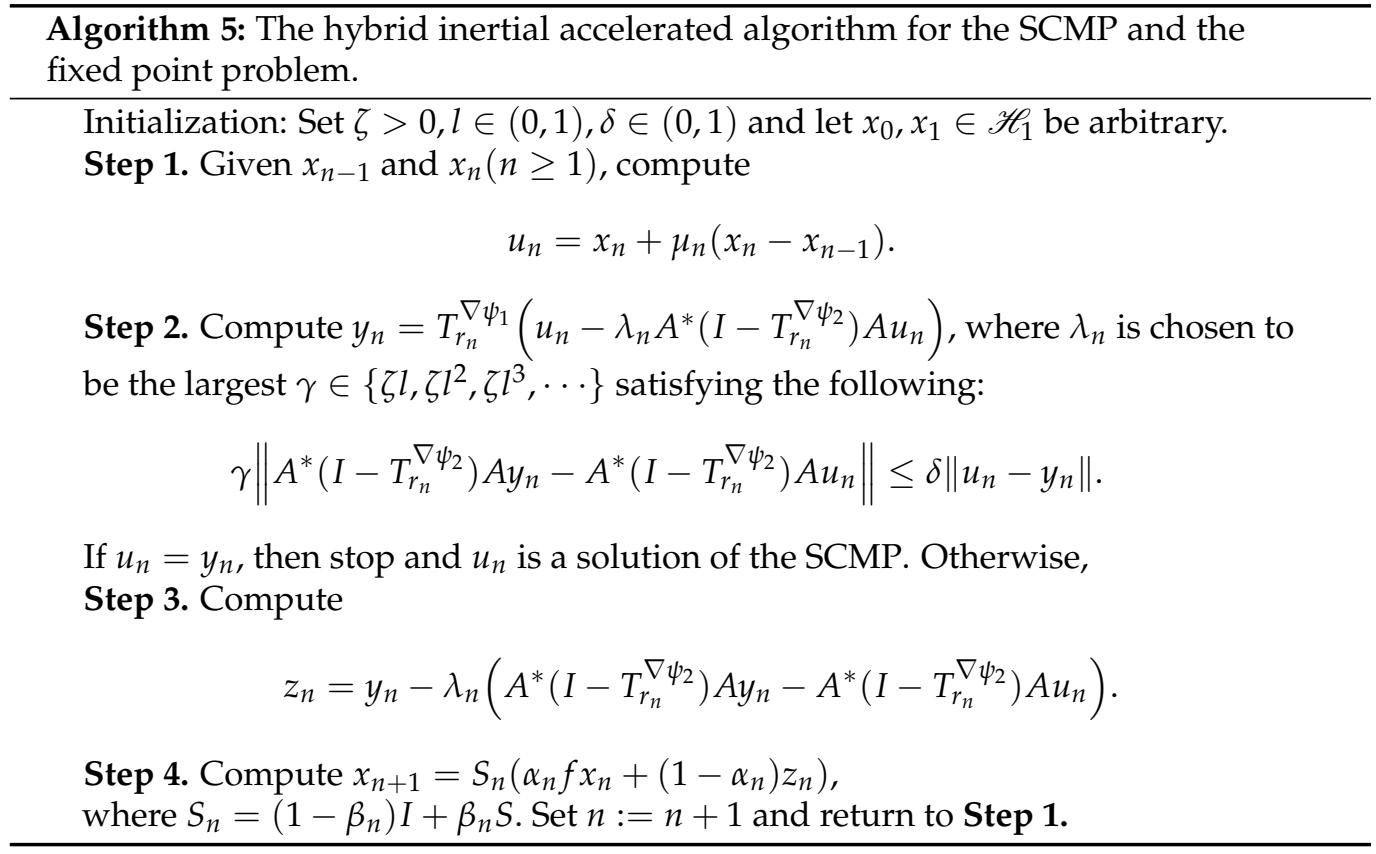

\section{Numerical Examples}

In this subsection, we discuss the numerical behavior of our proposed method in comparison with related methods in the literature. We compare Algorithm 3 with Algorithm 1 (Algorithm 3.1 of Jolaoso and Karahan [13]) and Algorithm 2 (Algorithm 2 of Cai et al. [17]).

Example 4. Let $\mathscr{H}_{1}=\mathscr{H}_{2}=\mathbb{R}$. Define $F_{1}: \mathbb{R} \times \mathbb{R} \rightarrow \mathbb{R}$ by $F_{1}(x, y)=-3 x^{2}+x y+2 y^{2}$ for all $x, y \in \mathbb{R}$ and $F_{2}: \mathbb{R} \times \mathbb{R} \rightarrow \mathbb{R}$ by $F_{2}(x, y)=-2 x^{2}+x y+y^{2}$ for all $x, y \in \mathbb{R}$. It is easy to observe that $F_{1}$ and $F_{2}$ satisfy (A1)-(A4). By Lemma 7 , it can be verified that $T_{r}^{F_{1}} x=\frac{x}{1+5 r}$ and $T_{r}^{F_{2}} x=\frac{x}{1+3 r}$. Let $f: \mathbb{R} \rightarrow \mathbb{R}$ be given by $f x=\frac{1}{2} x, A: \mathbb{R} \rightarrow \mathbb{R}$ be given by $A x=2 x$, and $S: \mathbb{R} \rightarrow \mathbb{R}$ be given by $S x=\frac{1}{3} x$ for all $x \in \mathbb{R}$. We can easily deduce that $f$ is a $\frac{1}{2}$-contraction, that $A$ is a bounded linear operator, and that $S$ is a 0 -demimetric mapping. Additionally, it is not difficult to check that Sol $=\{0\}$. Let us choose $\alpha_{n}=\frac{1}{2 n+1}, \beta_{n}=\frac{3}{4}, \mu_{n}=\frac{1}{2 n^{2}+n}, \eta=\frac{10}{13}$, and $\delta=\zeta=l=\frac{1}{2}$. We test Algorithms 1-3 for different values of $x_{0}$ and $x_{1}$ as follows:

Case I: $x_{0}=100$ and $x_{1}=80$;

Case II: $x_{0}=50$ and $x_{1}=35$;

Case III: $x_{0}=30$ and $x_{1}=25$;

Case IV: $x_{0}=10$ and $x_{1}=9$. 
We now compare the efficiency of Algorithm 3 with Algorithms 1 and 2. According to Figure 1, Tables 1-4, one finds that Algorithm 3 has a better convergence behavior than Algorithms 1 and 2.
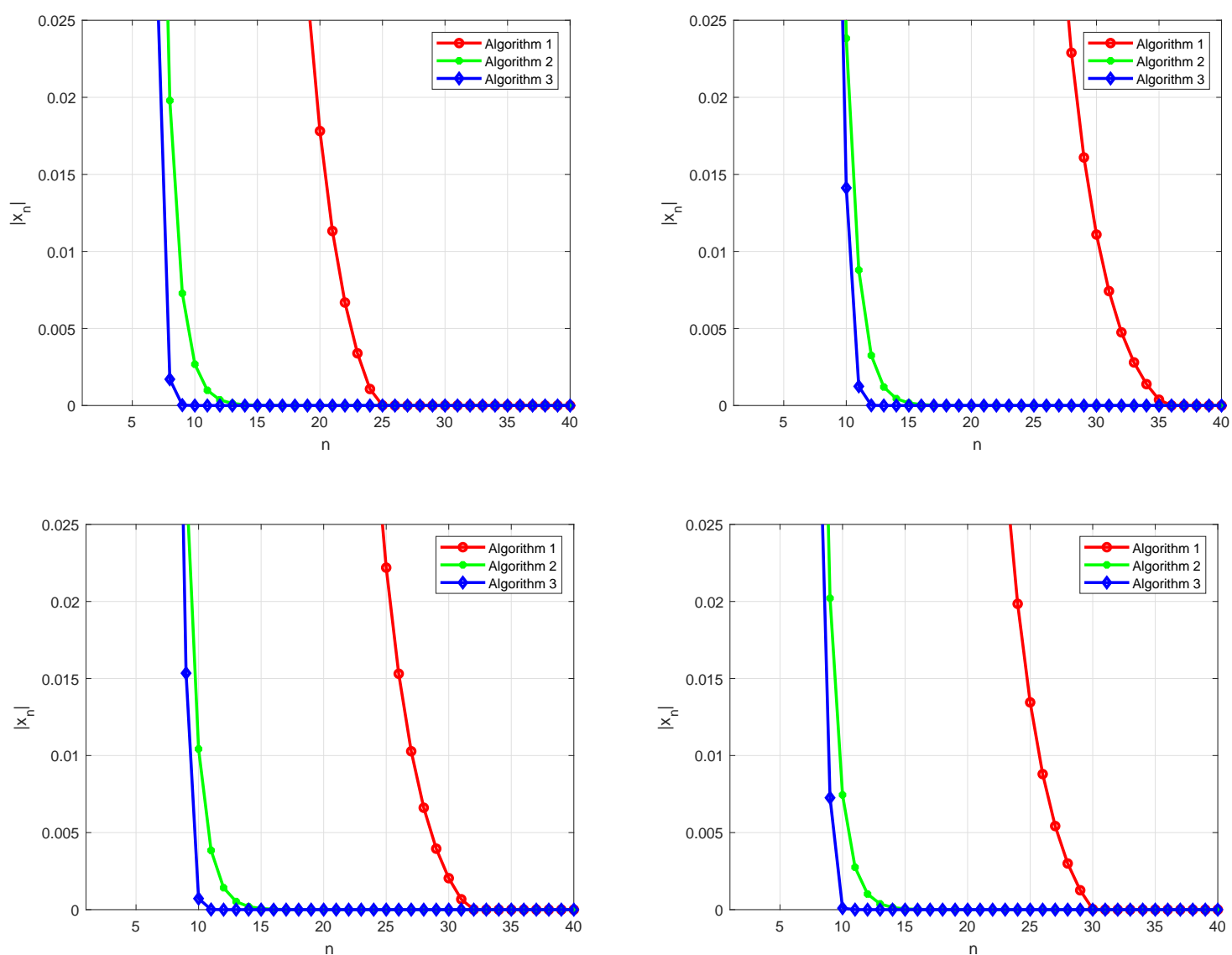

Figure 1. (Top left) Case I; (top right) Case II, (bottom left) Case III, (bottom right) Case IV.

Table 1. Numerical results for Example 4 in Case I.

\begin{tabular}{|c|c|c|c|c|c|c|}
\hline \multirow{2}{*}{ Err } & \multicolumn{2}{|l|}{ Algorithm 1} & \multicolumn{2}{|l|}{ Algorithm 2} & \multicolumn{2}{|l|}{ Algorithm 3} \\
\hline & $\left|x_{n}\right|$ & $n$ & $\left|x_{n}\right|$ & $n$ & $\left|x_{n}\right|$ & $n$ \\
\hline $10^{-3}$ & $1.0947111779190 \times 10^{-5}$ & 25 & $9.8932802287582 \times 10^{-4}$ & 11 & $2.5084018650195 \times 10^{-5}$ & 9 \\
\hline $10^{-4}$ & $1.0947111779190 \times 10^{-5}$ & 25 & $5.0103649467704 \times 10^{-5}$ & 14 & $2.5084018650195 \times 10^{-5}$ & 9 \\
\hline $10^{-5}$ & $1.0732462528617 \times 10^{-7}$ & 26 & $6.8899484598943 \times 10^{-6}$ & 16 & $3.3005287697625 \times 10^{-7}$ & 10 \\
\hline
\end{tabular}

Table 2. Numerical results for Example 4 in Case II.

\begin{tabular}{ccccccc}
\hline \multirow{2}{*}{ Err } & Algorithm 1 & \multicolumn{2}{c}{ Algorithm 2 } & \multicolumn{2}{c}{ Algorithm 3 } \\
\cline { 2 - 7 } & $\left|x_{n}\right|$ & $n$ & $\left|x_{n}\right|$ & $n$ & $\left|x_{n}\right|$ & $n$ \\
\hline $10^{-3}$ & $3.8495238046047 \times 10^{-4}$ & 36 & $5.5670721630783 \times 10^{-4}$ & 14 & $3.8440832718020 \times 10^{-5}$ & 12 \\
$10^{-4}$ & $2.6366601401402 \times 10^{-6}$ & 37 & $7.6554982887714 \times 10^{-5}$ & 16 & $3.8440832718020 \times 10^{-5}$ & 12 \\
$10^{-5}$ & $2.6366601401402 \times 10^{-6}$ & 37 & $3.9225807673322 \times 10^{-6}$ & 19 & $3.8440832718020 \times 10^{-7}$ & 13 \\
\hline
\end{tabular}


Table 3. Numerical results for Example 4 in Case III.

\begin{tabular}{ccccccc}
\hline \multirow{2}{*}{ Err } & \multicolumn{2}{c}{ Algorithm 1 } & \multicolumn{2}{c}{ Algorithm 2 } & \multicolumn{2}{c}{ Algorithm 3 } \\
\cline { 2 - 7 } & $\left|x_{n}\right|$ & $n$ & $\left|x_{n}\right|$ & $\boldsymbol{n}$ & $\left|x_{\boldsymbol{n}}\right|$ & $\boldsymbol{n}$ \\
\hline $10^{-3}$ & $6.7539211424883 \times 10^{-4}$ & 31 & $5.2608831941090 \times 10^{-4}$ & 13 & $7.1926227474796 \times 10^{-4}$ & 10 \\
$10^{-4}$ & $5.3602548749907 \times 10^{-6}$ & 32 & $7.2227962115800 \times 10^{-5}$ & 15 & $8.5626461279519 \times 10^{-6}$ & 11 \\
$10^{-5}$ & $5.3602548749907 \times 10^{-6}$ & 32 & $9.9463481554871 \times 10^{-6}$ & 17 & $8.5626461279519 \times 10^{-6}$ & 11 \\
\hline
\end{tabular}

Table 4. Numerical results for Example 4 in Case IV.

\begin{tabular}{ccccccc}
\hline \multirow{2}{*}{ Err } & Algorithm 1 & \multicolumn{2}{c}{ Algorithm 2 } & \multicolumn{2}{c}{ Algorithm 3 } \\
\cline { 2 - 7 } & $\left|x_{n}\right|$ & $n$ & $\left|x_{n}\right|$ & $n$ & $\left|x_{n}\right|$ & $n$ \\
\hline $10^{-3}$ & $3.0475500166712 \times 10^{-5}$ & 30 & $3.7577737100778 \times 10^{-4}$ & 13 & $9.5530385596659 \times 10^{-5}$ & 10 \\
$10^{-4}$ & $3.0475500166712 \times 10^{-5}$ & 30 & $5.1591401511286 \times 10^{-5}$ & 15 & $9.5530385596659 \times 10^{-5}$ & 10 \\
$10^{-5}$ & $2.4979918169436 \times 10^{-7}$ & 31 & $7.1045343967765 \times 10^{-6}$ & 17 & $1.1372664951983 \times 10^{-6}$ & 11 \\
\hline
\end{tabular}

\section{Conclusions}

In this paper, we study a new iterative algorithm for solving split equilibrium problems and fixed point problems of demimetric mappings in Hilbert spaces. A theorem of strong convergence was proven under some mild conditions. The numerical behavior of the new algorithm is also studied by reporting a numerical experiment. In particular, it can be seen that the suggested algorithm has competitive advantages over some existing methods.

Funding: This research was supported by the Key Scientific Research Project for Colleges and Universities in Henan Province (grant number 20A110038).

Institutional Review Board Statement: Not applicable.

Informed Consent Statement: Not applicable.

Data Availability Statement: All data in this research are available at request.

Acknowledgments: The authors thank the reviewers and the editors for their valuable comments for improving the original manuscript.

Conflicts of Interest: The author declare no conflict of interest.

\section{References}

1. Blum, E.; Oettli, W. From optimization and variational inequalities to equilibrium problems. Math. Stud. 1994, 63, 123-145.

2. Cho, Y.J.; Petrot, N.; Suantai, S. Fixed point theorems for nonexpansive mappings with applications to generalized equilibrium and system of nonlinear variational inequalities problems. J. Nonl. Anal. Opt. 2010, 1, 45-53.

3. Yao, Y.; Cho, Y.J.; Liou, Y.C. Algorithms of common solutions for variational inclusions, mixed equilibrium problems and fixed point problems. Eur. J. Oper. Res. 2011, 212, 242-250. [CrossRef]

4. Tan, B.; Qin, X.; Yao, J.C. Strong convergence of self-adaptive inertial algorithms for solving split variational inclusion problems with applications. J. Sci. Comput. 2021, 87, 1-34. [CrossRef]

5. Tseng, P. A modified forward-backward splitting method for maximal monotone mappings. SIAM J. Control Optim. 2000, 38, 431-446. [CrossRef]

6. Alakoya, T.O.; Jolaoso, L.O.; Mewomo, O.T. A general iterative method for finding common fixed point of finite family of demicontractive mappings with accretive variational inequality problems in Banach spaces. Nonlinear Stud. 2020, $27,1-24$.

7. Ogwo, G.N.; Izuchukwu, C.; Mewomo, O.T. Inertial methods for finding minimum-norm solutions of the split variational inequality problem beyond monotonicity. Numer. Algorithms 2021. [CrossRef]

8. Chidume, C.E.; Măruşter, Şt. Iterative methods for the computation of fixed points of demicontractive mappings. J. Comput. Appl. Math. 2010, 234, 861-882. [CrossRef]

9. Cai, G.; Dong, Q.-L.; Peng, Y. Strong convergence theorems for solving variational inequality problems with pseudo-monotone and non-Lipschitz operators. J. Opt. Theory Appl. 2021, 188, 447-472. [CrossRef]

10. Censor, Y.; Bortfeld, T.; Martin, B.; Trofimov, A. A unified approach for inversion problem in intensity-modulated radiation therapy. Phys. Med. Biol. 2006, 51, 2353-2365. [CrossRef] 
11. Ansari, Q.H.; Rehan, A. Split feasibility and fixed point problems. In Nonlinear Analysis: Approximation Theory, Optimization and Application; Ansari, Q.H., Ed.; Springer: New York, NY, USA, 2014; pp. 281-322.

12. Kazmi, K.R.; Rizvi, S.H. Iterative approximation of a common solution of a split equilibrium problem, a variational inequality problem and a fixed point problem. J. Egypt. Math. Soc. 2013, 21, 44-51. [CrossRef]

13. Jolaoso, L.O.; Karahan, I. A general alternative regularization method with line search technique for solving split equilibrium and fixed point problems in Hilbert spaces. Comput. Appl. Math. 2020, 39, 1-22. [CrossRef]

14. Alvarez, F.; Attouch, H. An inertial proximal method for monotone operators via discretization of a nonlinear oscillator with damping. Set-Valued Anal. 2001, 9, 3-11. [CrossRef]

15. Polyak, B.T. Introduction to Optimization; Optimization Software: New York, NY, USA, 1987.

16. Polyak, B.T. Some methods of speeding up the convergence of iterarive methods. Z. Vychisl. Mat. Mat. Fiz. 1964, 4, 1-17.

17. Cai, G.; Shehu, Y.; Iyiola, O.S. Inertial Tseng's extragradient method for solving variational inequality problems of pseudomonotone and non-Lipschitz operators. J. Ind. Manag. Optim. 2021. [CrossRef]

18. Takahashi, W. Introduction to Nonlinear and Convex Analysis; Yokohama Publishers: Yokohama, Japan, 2009.

19. Marino, G.; Xu, H.K. Weak and strong convergence theorems for strict pseudo-contractions in Hilbert spaces. J. Math. Anal. Appl. 2007, 329, 336-349. [CrossRef]

20. Takahashi, W. The split common fixed point problem and the shrinking projection method in banach spaces. J. Convex Anal. 2017, 24, 1015-1028.

21. Browder, F.E. Convergence of approximants to fixed points of nonexpansive nonlinear mappings in Banach spaces. Arch. Ration. Mech. Anal. 1967, 24, 82-90. [CrossRef]

22. Takahashi, W.; Wen, C.F.; Yao, J.C. The shrinking projection method for a finite family of demimetric mappings with variational inequality problems in a Hilbert space. Fixed Point Theory 2018, 19, 407-420. [CrossRef]

23. Song, Y. Iterative methods for fixed point problems and generalized split feasibility problems in Banach spaces. J. Nonl. Sci. Appl. 2018, 11, 198-217. [CrossRef]

24. Maingé, P.E. The viscosity approximation process for quasi-nonexpansive mappings in Hilbert spaces. Comput. Math. Appl. 2010, 59, 74-79. [CrossRef]

25. Xu, H.K. Iterative algorithm for nonlinear operators. J. Lond. Math. Soc. 2002, 2, 1-17. [CrossRef]

26. Combettes, P.L. Hirstoaga, S.A. Equilibrium programming in Hilbert spaces. J. Nonlinear Convex Anal. 2005, 6, 117-136.

27. Su, M.; Xu, H.-K. Remarks on the gradient-projection algorithm. J. Nonlinear Anal. Optim. 2010, 1, 35-43. 